\title{
3DEM Data Collection of Cancer Biopsies: Comparing FIB-SEM and SBF-SEM
}

Jessica L. Riesterer ${ }^{* 1,2}$, Kevin Loftis ${ }^{1}$, Kevin Stoltz ${ }^{1}$, Erin Stempinski ${ }^{1,2}$, Melissa Williams ${ }^{1.2}$, Claudia S. López $^{1,2,3}$, Guillaume Thibault ${ }^{1}$ and Joe W. Gray ${ }^{1}$

1. Oregon Health and Science University (OHSU), Center for Spatial Systems Biomedicine (OCSSB), Portland Oregon, USA.

2. Multiscale Microscopy Core, OHSU, Portland Oregon, USA.

3. Pacific Northwest Center for CryoEM, Portland Oregon, USA.

* Corresponding author: riesterj@ohsu.edu

Understanding cellular ultrastructure of cancer cells and the microenvironment within tumors is becoming increasingly important with respect to therapeutic targeting. Cell-cell interactions found in breast cancer have shown important signaling mechanisms occuring on filopodia-like protrusions that "reach out and touch" neighboring cells, potentially influencing cancer proliferation.[1] Nuclear invaginations observed in cultured cells indicate that cancer cells have a distinct nuclear morphology different from normal tissue.[2] Very recently, transcription factors within a cancer cell's nuclear membrane have been found to influence the cell's response to tumor microenviromental signals.[3]

Oregon Health and Science University (OHSU) is uniquely positioned to study cancer via model systems, such as cell culture, xenografts, and organoids. In addition, human subjects can be consented as part of the Brenden-Colson Center for Pancreatic Care, where pancreatic ductal adenocarcinoma (PDAC) patient tissue is collected via resection or biopsy and used for research purposes. Tissue samples are collected and immersed in Karnovsky's fixative (2.5\% Paraformaldehyde, $2.5 \%$ glutaraldehyde in $0.1 \mathrm{M}$ sodium cacodylate buffer) in the operating room for future evaluation of the tumor ultrastructure and microenvironment via electron microscopy.

Three-dimensional electron microscopy (3DEM) via scanning electron microscopy techniques (SEM) has recently become a mature technology within the life sciences.[4] The tumor behaviors described earlier are best understood in three-dimensions to truly model in vivo. 3DEM datasets have been collected via focused ion beam- and serial block face-scanning electron microscopy (FIB-SEM and SBF-SEM, respectively) on human PDAC specimens and compared for quality and usefulness. A FEI Helios NanoLab 660 DualBeam ${ }^{\mathrm{TM}}$ FIB-SEM and FEI Teneo VolumeScope ${ }^{\mathrm{TM}}$ SBF-SEM were used at low accelerating voltage (1-3 keV) to collect backscatter electron images serially and automated. Figures 1 and 2 show typical datasets manually annotated with Microscopy Image Browser [5] and reconstructed using Thermo Scientific's Amira ${ }^{\mathrm{TM}}$ software packages.

The two 3DEM techniques generate useful data at different length scales, but require different sample preparation and post-acquisition data handling. Data acquired via FIB-SEM are generally higher resolution (4-nm/voxel at best) and have better signal-to-noise, allowing easier feature annotation. Semiautomated segmentation approaches may be used with FIB-SEM data to decrease time-to-reconstruction. [6] FIB-SEM datasets are excellent for fine ultrastructure evaluation, such as nuclear arcitecture, presence of exo- or endocytic vesicles, and mitochondrion organization. SBF-SEM datasets are not capable of collecting high-resolution isotropic datasets (typically $10 \mathrm{~nm} \times 10 \mathrm{~nm}$ x 40nm voxels), making annotation difficult and semi-automatic rendering not possible due to the nature of the specimens (high volume of empty resin and irregular cellular ultrastructure.) In order to achieve low-noise images with SBF-SEM, 
post-fixation processing requires additional infiltration of heavy-metal species, even more so than FIBSEM requires, adding to processing time, and requires extra sample mounting/trimming steps. However, SBF-SEM produces larger volumes in the same time as FIB-SEM acquisitions that easily elucidate the tumor-microenvironment interactions. Cell behavior with surrounding stroma and immune cells is easily captured. In summary, 3DEM imaging technique choice should be thoughtfully considered due to the precious nature of cancer specimens [7].

\section{References:}

[1] M Creech et al., Scientific Reports [Online] 7, 40766 (2017), http://www.nature.com/articles/srep40766.

[2] D Jorgens et al., Journal of Cell Science 130 (2017), p. 177.

[3] Y Su et al., Genes \& Development 32 (2018), p. 1398.

[4] C Peddie and L Collinson, Micron 61 (2014), p. 9.

[5] I Belevich et al., PLoS Biology 14 (2016), p. e1002340.

https://doi.org/10.1371/journal.pbio.1002340. [6] K Loftis et al., Quantitative BioImaging (2019).

[7] Electron microscopy was performed at the Multiscale Microscopy Core (MMC) with technical support from the OHSU Center for Spatial Systems Biomedicine (OCSSB). The authors acknowledge funding from the OHSU Brenden-Colson Center for Pancreatic Care, the Prospect Creek Foundation and the OCSSB. Ms. Sharon Frase and Dr. Danielle Jorgens are thanked for discussions and contributions to this work.
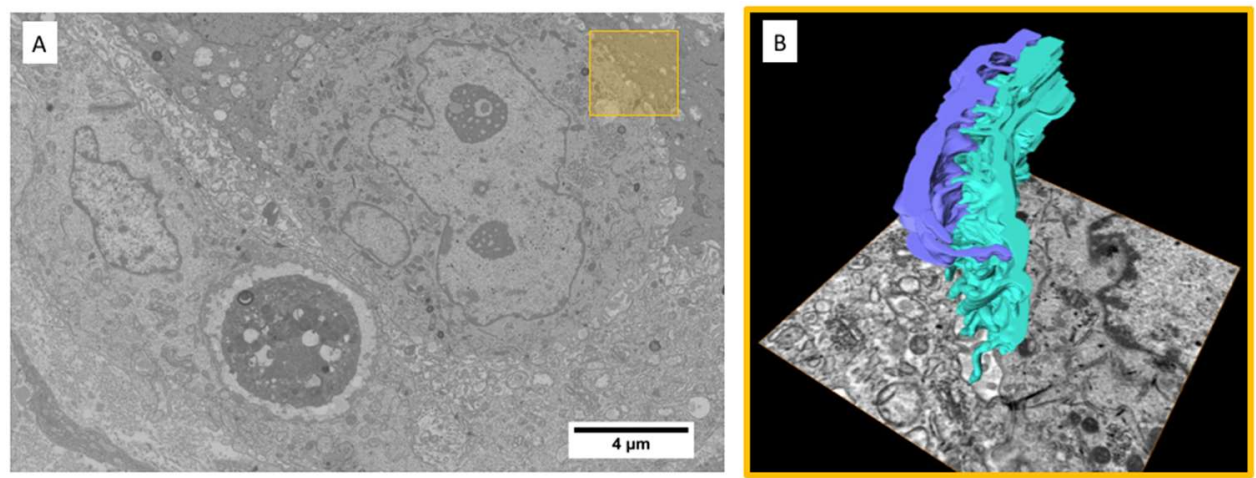

Figure 1. 3DEM Primary PDAC tumor tissue imaged using FIB-SEM. A) A single slice image of the dataset showing nuclear structure and cell-cell interactions. B) 3D rendering of cellular protrusions from A inset.
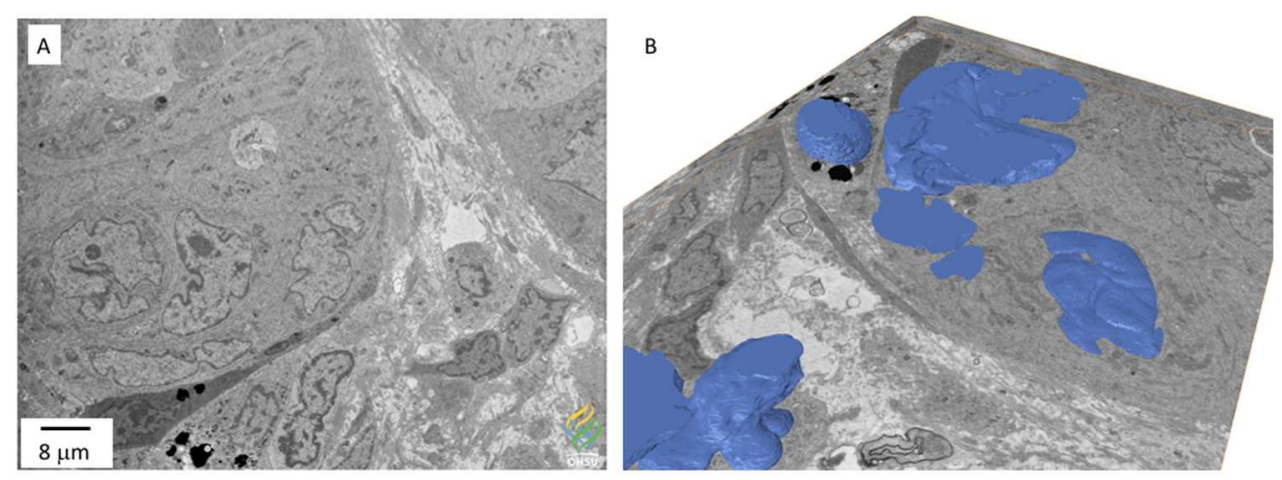

Figure 2. 3DEM PDAC metastasis to the liver imaged via SBF-SEM. A) A single slice image of the dataset showing stroma and microenvironment near several tumor cells. B) 3D rendering of cellular nuclei. 